\title{
SYMBOLIC EXTENSIONS FOR 3-DIMENSIONAL DIFFEOMORPHISMS
}

\author{
DAVID BURGUET * AND GANG LIAO **
}

\begin{abstract}
We prove that every $\mathcal{C}^{r}$ diffeomorphism with $r>1$ on a three-dimensional manifold admits symbolic extensions, i.e. topological extensions which are subshifts over a finite alphabet. This answers positively a conjecture of Downarowicz and Newhouse in dimension three.
\end{abstract}

\section{INTRODUCTION}

A symbolic extension of a topological dynamical system is a topological extension given by a subshift over a finite alphabet. Existence and entropy of symbolic extensions have been intensively investigated in the last decades. M. Boyle and T. Downarowicz [3] characterized the problem of existence in terms of new entropic invariants related to weak expansiveness properties of the system. In particular asymptotically $h$-expansive systems always admit principal symbolic extensions, i.e. extensions that preserve the entropy of invariant measures [4].

For smooth systems on compact manifolds this theory appears to be of highly interest. It is well known that Markov partitions allow to encode uniformly hyperbolic systems by finiteto-one symbolic extensions of finite type. Beyond uniform hyperbolicity, partially hyperbolic diffeomorphisms with one dimensional center satisfy the $h$-expansiveness property, hence admit principal symbolic extensions [12,18]. More recently the second author with M. Viana and J. Yang showed that smooth systems with no principal symbolic extension are $\mathcal{C}^{1}$-close to diffeomorphisms with homoclinic tangencies [17].

Moreover the existence of symbolic extensions depends on the order of smoothness. While $\mathcal{C}^{\infty}$ systems are asymptotically $h$-expansive $[8,24]$ and thus admit principal symbolic extensions, there is a $\mathcal{C}^{1}$ open set of 3 -dimensional diffeomorphisms [1] (resp. Lebesgue preserving diffeomorphisms $[9,15])$ in which generic ones have no symbolic extension. In intermediate smoothness, i.e. for $\mathcal{C}^{r}$ systems with $1<r<+\infty$, the existence was conjectured by T. Downarowicz and $\mathrm{S}$. Newhouse in [15] and in general this problem is still open. It has been first proved for circle maps by T. Downarowicz and A. Maass [14] and then by the first author for surface diffeomorphisms $[5,6]$. In this paper we work further on [6] to show existence of symbolic extensions for diffeomorphisms in dimension 3. We refer to the next section for the definitions and notations used in our main Theorem below.

Main Theorem. Let $f$ be a $\mathcal{C}^{r}$ diffeomorphism with $r>1$ on a compact 3-dimensional manifold $M$. Then $f$ admits a symbolic extension $\pi:(Y, S) \rightarrow(M, f)$ satisfying for all $\mu \in \mathcal{M}_{\text {inv }}(f)$ :

Date: November, 2019.

Key words and phrases. Symbolic extension; 3-dimensional diffeomorphism; tail entropy.

2010 Mathematics Subject Classification. 37B10, 37A35, 37C40.

${ }^{*}$ LPSM, Sorbonne Universite, Paris 75005 , France. ${ }^{* *}$ School of Mathematical Sciences, Center for Dynamical Systems and Differential Equations, Soochow University, Suzhou 215006, China; G. Liao was partially supported by NSFC (11701402, 11790274), BK 20170327 and IEPJ. 


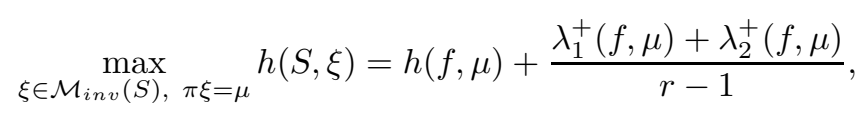

where $\lambda_{1}^{+}(f, \mu) \geq \lambda_{2}^{+}(f, \mu)$ denote the positive parts of the two largest Lyapunov exponents of $\mu$.

The ingredient of the present advance is mainly a new inequality relating the Newhouse local entropy of an ergodic measure and the local volume growth of smooth discs of unstable dimension (which is the number of positive Lyapunov exponents of the measure). Section 3 is devoted to the proof of this key estimate. Then for a 3-dimensional diffeomorphism, we may bound from above the Newhouse local entropy with respect to either $f$ or $f^{-1}$ by the local volume growth of curves, which implies the existence of symbolic extensions by combining with the Reparametrization Lemma developed in [6]. This is proved together with the Main Theorem in the last section.

\section{Preliminaries}

2.1. Newhouse entropy structure and the Symbolic Extension Theorem. Consider a topological system $(M, f)$, i.e. a continuous map $f: M \rightarrow M$ on a compact metric space $(M, d)$. For $x \in M, \varepsilon>0, n \in \mathbb{N}$, we denote the $n$-step dynamical ball at $x$ with radius $\varepsilon$ by

$$
B_{n}(x, \varepsilon, f)=\left\{y \in M: d\left(f^{i}(x), f^{i}(y)\right)<\varepsilon, i=0, \cdots, n-1\right\} .
$$

A subset $N$ of $M$ is said $(n, \delta)$-separated when any pair $y \neq z$ in $N$ satisfies $d\left(f^{i}(y), f^{i}(z)\right)>\delta$ for some $i \in[0, n-1]$. For any subset $\Lambda$ of $M$ and $\delta>0$, denote by $s(n, \delta, \Lambda)$ the maximal cardinality of the $(n, \delta)$-separated sets contained in $\Lambda$. For any $\Lambda \subset M, \varepsilon>0$, define

$$
h^{*}(f, \Lambda, \varepsilon)=\lim _{\delta \rightarrow 0} \limsup _{n \rightarrow \infty} \frac{1}{n} \log \sup _{x \in \Lambda} s\left(n, \delta, B_{n}(x, \varepsilon, f) \cap \Lambda\right) .
$$

Denote by $\mathcal{M}_{i n v}(f)\left(\right.$ resp. $\left.\mathcal{M}_{\text {erg }}(f)\right)$ the set of all $f$-invariant (resp. ergodic $f$-invariant) Borel probability measures endowed with the usual metrizable weak-* topology. Given $\mu \in \mathcal{M}_{\text {erg }}(f)$, for any $\varepsilon>0$, Newhouse [20] defined the tail entropy of $\mu$ at the scale $\varepsilon$ by letting

$$
h^{*}(f, \mu, \varepsilon)=\lim _{\eta \rightarrow 1,0<\eta<1} \inf _{\mu(\Lambda)>\eta} h^{*}(f, \Lambda, \varepsilon) .
$$

For $\mu \in \mathcal{M}_{i n v}(f)$, assuming $\mu=\int_{\mathcal{M}_{\text {erg }}(f)} \nu d M_{\mu}(\nu)$ is the ergodic decomposition of $\mu$, let

$$
h^{*}(f, \mu, \varepsilon)=\int_{\mathcal{M}_{\text {erg }}(f)} h^{*}(f, \nu, \varepsilon) d M_{\mu}(\nu) .
$$

Entropy structures are particular non-increasing sequences of nonnegative functions defined on $\mathcal{M}_{i n v}(f)$ which are converging pointwisely to the Kolmogorov-Sinai entropy function $h$ : $\mathcal{M}_{i n v}(f) \rightarrow \mathbb{R}^{+}$(see [13] for a precise definition). They satisfy the following criterion for the existence of symbolic extensions.

Theorem 1 (Symbolic Extension Theorem $[3,14])$. Let $(M, f)$ be a topological system. Assume $E$ is a nonnegative affine upper semicontinuous function such that for all $\mu \in \mathcal{M}_{\text {inv }}(f)$ there is an entropy structure $\left(h_{k}\right)_{k}$ satisfying

$$
\lim _{k} \limsup _{\mathcal{M}_{\text {erg }}(f) \ni \nu \rightarrow \mu}\left(E+h-h_{k}\right)(\nu) \leq E(\mu) .
$$


Then there exists a symbolic extension $\pi:(Y, S) \rightarrow(M, f)$ such that

$$
\max _{\xi \in \mathcal{M}_{i n v}(S),} h(S, \xi)=(E+h)(\mu) .
$$

Letting $\varepsilon_{k} \rightarrow 0$, then the sequence $\left(h_{k}^{N e w}\right)_{k}$ defined by $h_{k}^{N e w}(f, \mu):=h(f, \mu)-h^{*}\left(f, \mu, \varepsilon_{k}\right)$ for all $k \in \mathbb{N}$ and for all $\mu \in \mathcal{M}_{\text {inv }}(f)$ is an entropy structure [13]. As a matter of fact, for any $m \in \mathbb{Z} \backslash\{0\}, h_{m, k}^{N e w}(f, \mu):=h(f, \mu)-\frac{1}{|m|} h^{*}\left(f^{m}, \mu, \varepsilon_{k}\right)$ for all $k \in \mathbb{N}$ and for all $\mu \in \mathcal{M}_{\text {inv }}(f)$ is also an entropy structure (see Lemma 1 in [6]).

2.2. Lyapunov exponents. Let $f: M \rightarrow M$ be a differentiable map on a compact Riemannian manifold $(M,\|\cdot\|)$ of dimension $\mathrm{d}$. Given $x \in M$, the Lyapunov exponent relative to a direction $v \in T_{x} M$ is the exponential growth rate given by the limit

$$
\lim _{n \rightarrow \infty} \frac{1}{n} \log \left\|D_{x} f^{n} v\right\|
$$

which exists for almost every point $x$ with respect to every $f$-invariant Borel probability measure $\mu$ by Oseledets theorem [21] (it does not depend on the Riemannian structure on $M$ ). Moreover, for $\mu$-almost every point $x$, there exist values $\lambda_{1}(f, x) \geq \cdots \geq \lambda_{\mathrm{d}}(f, x)$ of the limit (2) and measurable flags of the tangent spaces $\{0\}=G_{x}^{\mathrm{d}+1} \subset G_{x}^{\mathrm{d}} \subset \cdots \subset G_{x}^{1}=T_{x} M$ satisfying:

$$
\lim _{n \rightarrow \infty} \frac{1}{n} \log \left\|D_{x} f^{n} v\right\|=\lambda_{i}(f, x), \quad \forall v \in G_{x}^{i} \backslash G_{x}^{i+1}, 1 \leq i \leq \mathrm{d} .
$$

For any $\mu \in \mathcal{M}_{\text {inv }}(f), 1 \leq i \leq \mathrm{d}$, we denote

$$
\begin{array}{r}
\lambda_{i}(f, \mu)=\int \lambda_{i}(f, x) d \mu(x), \\
\sum_{j=1}^{i} \lambda_{j}^{+}(f, \mu)=\int \sum_{j=1}^{i} \lambda_{j}^{+}(f, x) d \mu(x) .
\end{array}
$$

For $\nu \in \mathcal{M}_{\text {erg }}(f)$, we have $\lambda_{i}(f, \nu)=\lambda_{i}(f, x)$ for all $i$ and for $\nu$-almost every $x$. By standard arguments the function $\mu \mapsto \sum_{j=1}^{i} \lambda_{j}^{+}(f, \mu)$ defines an affine upper semicontinuous function on $\mathcal{M}_{i n v}(f)$ (see Lemma 3 in [6]). For a $\mathcal{C}^{r}$ diffeomorphism with $r>1$ on a compact 3-dimensional Riemannian manifold, we will prove that $E=\frac{\sum_{j=1}^{2} \lambda_{j}^{+}(f, \cdot)}{r-1}$ satisfies Inequality (1), which together with Theorem 1 implies the Main Theorem.

2.3. Nonuniformly hyperbolic estimates. Assume now $f$ is a diffeomorphism. In this case, Oseledets theorem provides for any $\mu \in \mathcal{M}_{\text {inv }}(f)$, for $\mu$-a.e., $x \in M$, a decomposition on the tangent space $T_{x} M=E_{x}^{c s} \oplus E_{x}^{u}$ and $\rho_{c s}(x) \leq 0<\rho_{u}(x)$ satisfying

- $\lim _{|n| \rightarrow \infty} \frac{1}{n} \log \left\|D_{x} f^{n}(v)\right\| \leq \rho_{c s}(x), \quad \forall 0 \neq v \in E_{x}^{c s}$

- $\lim _{|n| \rightarrow \infty} \frac{1}{n} \log \left\|D_{x} f^{n}(w)\right\| \geq \rho_{u}(x), \quad \forall 0 \neq w \in E_{x}^{u}$;

- $\lim _{|n| \rightarrow \infty} \frac{1}{n} \log \sin \angle\left(E_{f^{n}(x)}^{c s}, E_{f^{n}(x)}^{u}\right)=0$.

For $0<\gamma \ll \lambda_{u}$ and $k \in \mathbb{N}$, we consider the sets $\Lambda_{k}\left(\lambda_{u}, \gamma\right)$ consisting of points $x$ in $M$ with the following properties: 
- $\left\|D f^{n} \mid E_{f^{i}(x)}^{c s}\right\| \leq e^{k \gamma} e^{|i| \gamma} e^{n \gamma}, \quad \forall i \in \mathbb{Z}, n \geq 1$;

- $\left\|D f^{-n} \mid E_{f^{i}(x)}^{u}\right\| \leq e^{k \gamma} e^{|i| \gamma} e^{n\left(-\lambda_{u}+\gamma\right)}, \quad \forall i \in \mathbb{Z}, n \geq 1$;

- $\sin \angle\left(E_{f^{i}(x)}^{c s}, E_{f^{i}(x)}^{u}\right) \geq e^{-k \gamma} e^{-|i| \gamma}, \quad \forall i \in \mathbb{Z}$.

From the definition, it holds that [2, 22]

- $T_{x} M=E_{x}^{c s} \oplus E_{x}^{u}$ is a continuous splitting on each $\Lambda_{k}\left(\lambda_{u}, \gamma\right)$;

- $f^{ \pm}\left(\Lambda_{k}\left(\lambda_{u}, \gamma\right)\right) \subset \Lambda_{k+1}\left(\lambda_{u}, \gamma\right)$ for any $k \in \mathbb{N}$;

- $x \in \bigcup_{k \in \mathbb{N}} \Lambda_{k}\left(\lambda_{u}, \gamma\right)$ provided $\lambda_{u} \leq \rho_{u}(x)$;

- $\lim _{\lambda_{u} \rightarrow 0} \mu\left(\bigcup_{k \in \mathbb{N}} \Lambda_{k}\left(\lambda_{u}, \gamma\right)\right)=1$ for any $\mu \in \mathcal{M}_{i n v}(f)$.

For the sake of statements, we let $\Lambda_{k}=\Lambda_{k}\left(\lambda_{u}, \gamma\right)$ for any $k \in \mathbb{N}$ and $\Lambda^{*}=\bigcup_{k \in \mathbb{N}} \Lambda_{k}\left(\lambda_{u}, \gamma\right)$. Denote $\lambda_{u}^{\prime}=\lambda_{u}-2 \gamma$. Given $x \in \Lambda^{*}$, define for all $v=v_{c s}+v_{u}$ and $w=w_{c s}+w_{u}$ with $v_{c s}, w_{c s} \in E_{x}^{c s}$ and $v_{u}, w_{u} \in E_{x}^{u}$,

$$
\begin{aligned}
<v_{c s}, w_{c s}>^{\prime} & =\sum_{n=0}^{+\infty} e^{-4 n \gamma}<D_{x} f^{n}\left(v_{c s}\right), D_{x} f^{n}\left(w_{c s}\right)> \\
<v_{u}, w_{u}>^{\prime} & =\sum_{n=0}^{+\infty} e^{2 n \lambda_{u}^{\prime}}<D_{x} f^{-n}\left(v_{u}\right), D_{x} f^{-n}\left(w_{u}\right)> \\
<v, w>^{\prime} & =<v_{u}, w_{u}>^{\prime}+<v_{c s}, w_{c s}>^{\prime} .
\end{aligned}
$$

There exists $a_{1}=a_{1}(\gamma)>1$ such that

$$
\|v\| \leq\|v\|^{\prime} \leq a_{1} e^{k \gamma}\|v\|, \quad \forall v \in T_{\Lambda_{k}} M .
$$

The norm $\|\cdot\|^{\prime}$ is called a Lyapunov metric, with which $f$ behaves uniformly on $\Lambda^{*}$ :

$$
\begin{aligned}
& \frac{1}{\left\|D f^{-1}\right\|}\left\|v_{c s}\right\|_{x}^{\prime} \leq\left\|D_{x} f\left(v_{c s}\right)\right\|_{f(x)}^{\prime} \leq e^{2 \gamma}\left\|v_{c s}\right\|_{x}^{\prime}, \\
& \frac{1}{\|D f\|}\left\|v_{u}\right\|_{x}^{\prime} \leq\left\|D_{x} f^{-1}\left(v_{u}\right)\right\|_{f^{-1}(x)}^{\prime} \leq e^{-\lambda_{u}^{\prime}}\left\|v_{u}\right\|_{x}^{\prime} .
\end{aligned}
$$

In this manner, the splitting $T_{\Lambda^{*}} M=E^{c s} \oplus E^{u}$ is dominated with respect to \|\|$^{\prime}$, i.e.

$$
\begin{gathered}
\frac{\left\|D_{x} f\left(v_{c s}\right)\right\|^{\prime}}{\left\|D_{x} f\left(v_{u}\right)\right\|^{\prime}} \leq e^{2 \gamma-\lambda_{u}^{\prime}} \frac{\left\|v_{c s}\right\|^{\prime}}{\left\|v_{u}\right\|^{\prime}}, \quad \forall 0 \neq v_{c s} \in E_{x}^{c s}, 0 \neq v_{u} \in E_{x}^{u}, x \in \Lambda^{*}, \\
\text { with } 2 \gamma-\lambda_{u}^{\prime}<0 .
\end{gathered}
$$

We consider a $\mathcal{C}^{r}$ diffeomorphism $f$ on a $C^{r}$ smooth Riemanian manifold $\left.(M,\|\cdot\|)\right)$ with $r>1$. Let $\alpha=\min \{r-1,1\}$. We are going to state that the dominated behavior on each $\Lambda_{k}$ can be extended to a $e^{-k \mathrm{~d} \gamma^{\prime}}$-neighborhood for $\gamma^{\prime}=\alpha^{-1} \gamma$. Moreover, for attaining a preassigned 
local proximity of dominated splitting, we may choose a positive number $b$ independently of $k$ such that this proximity holds in a $b e^{-k \mathrm{~d} \gamma^{\prime}}$-neighborhood of $\Lambda_{k}$.

Let $d$ be the Riemannian distance on $M$ and $r$ be the radius of injectivity of $(M,\|\cdot\|)$. The ball at $x \in M$ of radius $R \in \mathbb{R}^{+}$with respect to $d$ is denoted by $B(x, R)$. Then for $y \in B(x, \mathrm{r})$ we use the identification

$$
\begin{aligned}
T_{B(x, \mathrm{r})} M & \simeq B(x, \mathrm{r}) \times T_{x} M \\
(y, v) & \mapsto\left(y, D_{y}\left(\exp _{x}^{-1}\right)(v)\right)
\end{aligned}
$$

to "translate" the vector $v \in T_{y} M$ to the vector $\hat{v}_{x}:=D_{y}\left(\exp _{x}^{-1}\right)(v) \in T_{x} M$. Recall that the exponential map $(x, v) \mapsto \exp _{x}(v)$ defines a $C^{r}$ map (thus $C^{1+\alpha}$ ) from $T M$ to $M$ with $D_{x}\left(\exp _{x}\right)=\operatorname{Id}_{T_{x} M}$. Since the diffeomorphism $f$ is also $C^{1+\alpha}$ on the compact manifold $M$, there exist $K>1, a_{2}>0$ such that

$$
\begin{array}{cc}
\forall x \in M, \forall(y, v) \in T_{B\left(x, a_{2}\right)} M, & \frac{\|v\|}{2} \leq\left\|\hat{v}_{x}\right\| \leq 2\|v\| \\
\text { and }\left\|D_{x} f^{ \pm}\left(\hat{v}_{x}\right)-\widehat{D_{y} f^{ \pm}} v_{f(x)}\right\| \leq K\|v\| d(x, y)^{\alpha} .
\end{array}
$$

For $x \in \Lambda^{*}$ and $(y, v) \in T_{B\left(x, a_{2}\right)} M$, we define $\|v\|_{x}^{\prime \prime}=\left\|\hat{v}_{x}\right\|^{\prime}$ and we also let $<,>_{x}^{\prime \prime}$ be the associated scalar product on $T_{y} M$. It follows then from (3) that

$$
\forall x \in \Lambda_{k}, \forall(y, v) \in T_{B\left(x, a_{2}\right)} M, \quad 2 a_{1} e^{k \gamma}\|v\| \geq\|v\|_{x}^{\prime \prime}=\left\|\hat{v}_{x}\right\|^{\prime} \geq \frac{\|v\|}{2} .
$$

We write $\hat{v}_{x}$ as $v$ whenever there is no confusion and we also denote by $T_{y} M=E_{x}^{c s} \oplus E_{x}^{u}$ the splitting of $T_{y} M$ which translates to the splitting $T_{x} M=E_{x}^{c s} \oplus E_{x}^{u}$. Let $\lambda_{u}^{\prime \prime}=\lambda_{u}^{\prime}-\gamma$ and let $a_{2}^{\prime}>0$ such that $f^{i}\left(B\left(x, a_{2}^{\prime}\right)\right) \subset B\left(f^{i} x, a_{2}\right)$ for all $x \in M$ and $i=0,1,-1$. Then define

$$
\gamma_{k}=\min \left\{1, a_{2}^{\prime},\left(\frac{e^{-\lambda_{u}^{\prime \prime}}-e^{-\lambda_{u}^{\prime}}}{4 a_{1} e^{(k+1) \gamma} K}\right)^{\frac{1}{\alpha}}\right\} .
$$

Then we have for all $x \in \Lambda_{k}$, for all $y \in B\left(x, \gamma_{k}\right)$ and for all $v_{c s / u} \in E_{x}^{c s / u} \subset T_{y} M$ (see [22] p.72 for further details) :

$$
\begin{gathered}
\left(\frac{1}{\left\|D f^{-1}\right\|}-\left(e^{\gamma}-1\right)\right)\left\|v_{c s}\right\|_{x}^{\prime \prime} \leq\left\|D_{y} f\left(v_{c s}\right)\right\|_{f(x)}^{\prime \prime} \leq e^{3 \gamma}\left\|v_{c s}\right\|_{x}^{\prime \prime}, \\
\left(\frac{1}{\|D f\|}-\left(e^{\gamma}-1\right)\right)\left\|v_{u}\right\|_{x}^{\prime \prime} \leq\left\|D_{y} f^{-1}\left(v_{u}\right)\right\|_{f^{-1}(x)}^{\prime \prime} \leq e^{-\lambda_{u}^{\prime \prime}}\left\|v_{u}\right\|_{x}^{\prime \prime} .
\end{gathered}
$$

Define $\kappa(x)=\min \left\{k \in \mathbb{N}: x \in \Lambda_{k}\right\}$ for $x \in \Lambda^{*}$. Then the inequalities (5) and (6) hold for any $y \in B\left(x, \gamma_{\kappa(x)}\right)$. Such sets $B\left(x, \gamma_{\kappa(x)}\right)$ are called Lyapunov neighborhoods. Letting $\gamma^{\prime}=\alpha^{-1} \gamma$, we have $\gamma_{k}=a_{3} e^{-k \gamma^{\prime}}<1$ for $k$ large enough and some constant $a_{3}$ independent of $k$. We use $d_{x}^{\prime \prime}$ to denote the distance induced by $\|\cdot\|_{x}^{\prime \prime}$ on $B\left(x, a_{2}\right)$ and $B_{x}^{\prime \prime}(y, r)$ to denote the ball centered at $y$ with radius $r$ in $d_{x}^{\prime \prime}$.

For the purpose of our use in the computation of tail entropy and local volume growth, we need to estimate the proximity of the dominated splitting in Lyapunov neighborhoods along orbits. For a splitting $F=F_{1} \oplus F_{2}$ of an Euclidean space $F$ with norm \|\| , and $\xi>0$, we denote by $Q_{\|\|}\left(F_{1}, \xi\right)$ the cone of width $\xi$ of $F_{1}$ in \|\| , i.e. the set $\left\{v=v_{1}+v_{2} \in F: v_{1} \in F_{1}, v_{2} \in\right.$ $\left.F_{2}, \quad\left\|v_{2}\right\| \leq \xi\left\|v_{1}\right\|\right\}$. For any vector subspace $G$ of $F$ we let $\iota(G)$ be the Plücker embedding of $G$ in the projective space $\mathbb{P} \wedge F$ of the Euclidean power exterior algebra $\curlywedge F$. When $A: F \rightarrow F^{\prime}$ is a linear map between two finite dimensional Euclidean spaces $F$ and $F^{\prime}$, we let $\wedge^{l} A$ be the 
induced map on the $l$-exterior power $\Lambda^{l} F$ with $l$ less than or equal to the dimension of $F$. With the above notations the map $x \mapsto \wedge^{l} D_{x} f$ is $\alpha$-Hölder and one may assume its Hölder norm is less than $K$ by taking $K$ larger in advance. Observe that $\Lambda^{l} F \ni u \mapsto\left\|\Lambda^{l} A u\right\|$ induces a map on $\mathbb{P} \wedge^{l} F$ by letting $\left\|\wedge^{l} A(\mathbb{P} u)\right\|=\frac{\left\|\Lambda^{l} A u\right\|}{\|u\|}$. Also we let $l_{u}(f, z)$ be the dimension of $E^{u}(z)$. When $\mu \in \mathcal{M}_{\text {erg }}(f), l_{u}(f, z)$ is a constant for $\mu$-a.e. $z$, which we denote by $l_{u}(f, \mu)$.

Lemma 1. For any $\xi>0$ small enough there exists $a_{\xi}>0$ such that for any $x \in \Lambda^{*}$ and for any $y \in B\left(x, a_{\xi} \gamma_{\kappa(x)}^{l}\right)$ with $l=l_{u}(f, x)$ we have :

(i) $\left\|D_{y} f(v)\right\|_{f(x)}^{\prime \prime} \geq e^{\lambda_{u}^{\prime \prime}-\gamma}\|v\|_{x}^{\prime \prime}$ for all $v \in Q_{\|\|_{x}^{\prime \prime}}\left(E_{x}^{u}, \xi\right)$ and $\left\|D_{y} f(v)\right\|_{f(x)}^{\prime \prime} \leq e^{4 \gamma}\|v\|_{x}^{\prime \prime}$ for all $v \in Q_{\|\|_{x}^{\prime \prime}}\left(E_{x}^{c s}, \xi\right)$

(ii) $D_{y} f\left(Q_{\|\|_{x}^{\prime \prime}}\left(E_{x}^{u}, \xi\right)\right) \subset Q_{\|\|_{f(x)}^{\prime \prime}}\left(E_{f(x)}^{u}, \xi\right)$ and $D_{y} f^{-1}\left(Q_{\|\|_{x}^{\prime \prime}}\left(E_{x}^{c s}, \xi\right)\right) \subset Q_{\|\|_{f}^{\prime \prime}(x)}^{\prime \prime}\left(E_{f^{-1}(x)}^{c s}, \xi\right)$,

(iii) $e^{-\gamma} \leq \frac{\left\|\lambda^{l} D_{y} f(\iota(G))\right\|_{f(x)}^{\prime \prime}}{\left\|\lambda^{l} D_{x} f\left(\iota\left(E_{x}^{u}\right)\right)\right\|_{f(x)}^{\prime \prime}} \leq e^{\gamma}$ for all l-plane $G \subset Q_{\|\|_{x}^{\prime \prime}}\left(E_{x}^{u}, \xi\right)$.

Proof. Let $\xi>0$ and $x \in \Lambda^{*}$.

(i) By the domination property $E_{x}^{c s} \oplus E_{x}^{u}$ at $y$ with respect to $\|\cdot\|_{x}^{\prime \prime}$ given by the inequalities (5) and (6), the first item holds for small $\xi$ independent of $\kappa(x)$.

(ii) Using the invariance of $E^{u}$ and the domination property at $x$ there exists $\varsigma \in(0,1)$ independent of $x$ satisfying $D_{x} f\left(Q_{\|\|_{x}^{\prime \prime}}\left(E_{x}^{u}, \xi\right)\right) \subset Q_{\|\|_{f(x)}^{\prime \prime}}\left(E_{f(x)}^{u}, \varsigma \xi\right)$. Then for any $y \in$ $B\left(x, a_{\xi} \gamma_{\kappa(x)}\right)$, we get by the Inequalities (4)

$$
\begin{aligned}
\left\|D_{x} f-D_{y} f\right\|_{x}^{\prime \prime} & :=\max _{\|v\|_{x}^{\prime \prime}=1}\left\|D_{x} f(v)-D_{y} f(v)\right\|_{f(x)}^{\prime \prime} \\
& \leq 4 a_{1} e^{\kappa(f(x)) \gamma}\left\|D_{x} f-D_{y} f\right\| \\
& \leq 4 K a_{1} e^{(\kappa(x)+1) \gamma}\left(a_{\xi} \gamma_{\kappa(x)}\right)^{\alpha} \\
& \leq a_{\xi}^{\alpha} .
\end{aligned}
$$

For small $\gamma$, by (5) and (6) one has also

$$
\frac{1}{2\left\|D f^{-1}\right\|} \leq \min _{\|v\|_{x}^{\prime \prime}=1}\left\|D_{y} f(v)\right\|_{f(x)}^{\prime \prime} \leq \max _{\|v\|_{x}^{\prime \prime}=1}\left\|D_{y} f(v)\right\|_{f(x)}^{\prime \prime} \leq 2\|D f\| .
$$

It follows that for $\|v\|_{x}^{\prime \prime}=1$, the angle $L^{\prime \prime}\left(D_{y} f(v), D_{x} f(v)\right)$ with respect to $\|\cdot\|_{f(x)}^{\prime \prime}$ is less than $\arctan (\xi)-\arctan (\varsigma \xi)$ for $a_{\xi}$ small enough. We conclude that $D_{y} f\left(Q_{\|\|_{x}^{\prime \prime}}\left(E_{x}^{u}\right), \xi\right) \subset$ $Q_{\|\|_{f(x)}^{\prime \prime}}\left(E_{f(x)}^{u}, \xi\right)$ for any $y \in B\left(x, a_{\xi} \gamma_{k}\right)$. We prove similarly the cone invariance property for the center stable direction.

(iii) To prove the last item observe first that using again the domination property at $x$ we get $\left|\frac{\left\|\mathcal{\curlywedge}^{l} D_{x} f(\iota(G))\right\|_{f(x)}^{\prime \prime}}{\left\|\mathcal{\curlywedge}^{\imath} D_{x} f\left(\iota\left(E_{x}^{u}\right)\right)\right\|_{f(x)}^{\prime \prime}}-1\right| \leq 1-e^{-\gamma / 2}$ for all $l$-planes $G \subset Q_{\|\|_{x}^{\prime \prime}}\left(E_{x}^{u}, \xi\right)$ for $\xi>0$ small 


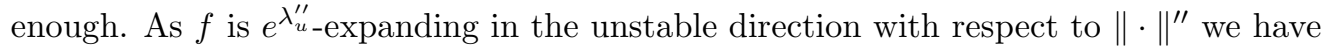

$$
\left\|\wedge^{l} D_{x} f\left(\iota\left(E_{x}^{u}\right)\right)\right\|_{f(x)}^{\prime \prime} \geq e^{l \lambda_{u}^{\prime \prime}} .
$$

Then arguing as above for $y \in B\left(x, a_{\xi} \gamma_{\kappa(x)}^{l}\right)$, we have by Lemma 2 in the Appendix and the Inequalities (4):

$$
\left\|\curlywedge^{l} D_{x} f-\Lambda^{l} D_{y} f\right\|_{f(x)}^{\prime \prime} \leq\left(4 a_{1} e^{\kappa(f(x)) \gamma}\right)^{l}\left\|\curlywedge^{l} D_{x} f-\curlywedge^{l} D_{y} f\right\| \leq a_{\xi}^{\alpha} .
$$

Therefore we get for $a_{\xi}$ small enough :

$$
\begin{aligned}
\left|\frac{\left\|\Lambda^{l} D_{y} f(\iota(G))\right\|_{f(x)}^{\prime \prime}}{\left\|\Lambda^{l} D_{x} f\left(\iota\left(E_{x}^{u}\right)\right)\right\|_{f(x)}^{\prime \prime}}-1\right| & \leq \frac{\left\|\Lambda^{l} D_{y} f(\iota(G))\right\|-\left\|\Lambda^{l} D_{x} f(\iota(G))\right\|_{f(x)}^{\prime \prime} \mid}{\left\|\Lambda^{l} D_{x} f\left(\iota\left(E_{x}^{u}\right)\right)\right\|_{f(x)}^{\prime \prime}} \\
& +\left|\frac{\left\|\Lambda^{l} D_{x} f(\iota(G))\right\|_{f(x)}^{\prime \prime}}{\left\|\wedge^{l} D_{x} f\left(\iota\left(E_{x}^{u}\right)\right)\right\|_{f(x)}^{\prime \prime}}-1\right| \\
& \leq \frac{a_{\xi}^{\alpha}}{e^{l \lambda_{u}^{\prime \prime}}}+1-e^{-\gamma / 2} \\
& \leq 1-e^{-\gamma} .
\end{aligned}
$$

From the domination structure $E^{c s} \oplus E^{u}$ in the norm $\|\cdot\|_{x}^{\prime \prime}$, one may build a family of fake center-stable manifolds as follows.

Proposition 1. With the notations of Lemma 1, for any $\xi>0$ small enough, there exist $b_{\xi} \in\left(0, a_{\xi}\right)$ and families $\left\{\mathcal{W}_{x}^{c s}: x \in \Lambda^{*}\right\}$ of $C^{1}$ manifolds satisfying

(i) uniform size: for $x \in \Lambda_{k}, k \in \mathbb{N}$, there is a $C^{1}$ map $\phi_{x}: E_{x}^{c s} \rightarrow E_{x}^{u}$ such that $\mathcal{W}_{x}^{c s}$ is locally given by the graph $\Gamma \phi_{x}:=\left\{\left(z, \phi_{x}(z)\right), z \in E_{x}^{c s}\right\}$ of $\phi_{x}$, i.e.

$$
\mathcal{W}_{x}^{c s}=\exp _{x}\left(\Gamma \phi_{x}\right) \cap B\left(x, a_{\xi} \gamma_{k}\right)
$$

(ii) almost tangency: $T_{y} \mathcal{W}_{x}^{c s}$ lies in a cone of width $\xi$ of $E_{x}^{c s}$ in $\|\cdot\|_{x}^{\prime \prime}$ for any $y \in \mathcal{W}_{x}^{c s}$;

(iii) local invariance: $f^{ \pm} \mathcal{W}_{x}^{c s}\left(b_{\xi} \gamma_{\kappa(x)}\right) \subset \mathcal{W}_{f^{ \pm}(x)}^{c s}$ with $\mathcal{W}_{x}^{c s}(\zeta)$ being the ball of radius $\zeta$ centered at $x$ inside $\mathcal{W}_{x}^{c s}$ with respect to the distance induced by $\|\cdot\|_{x}^{\prime \prime}$ on $\mathcal{W}_{x}^{c s}$.

Proof. By taking the exponential map at $x$ we can assume without loss of generality that we are working in $\mathbb{R}^{\mathrm{d}}$. Let $\xi$ and $a_{\xi}$ be as in Lemma 1. For any $x \in \Lambda^{*}$, we can extend $\left.f\right|_{B\left(x, a_{\xi} \gamma_{\kappa(x)}\right)}$ to a diffeomorphism $\tilde{f}_{x}: \mathbb{R}^{\mathrm{d}} \rightarrow \mathbb{R}^{\mathrm{d}}$ such that

- $\tilde{f}_{x}(y)=f(y)$ for $y \in B\left(x, a_{\xi} \gamma_{\kappa(x)}\right)$;

- $\left\|D_{y} \tilde{f}_{x}-D_{x} f\right\|_{x}^{\prime \prime} \leq 2 a_{\xi}^{\alpha} \quad$ for $y \in \mathbb{R}^{\mathrm{d}}$.

By taking $a_{\xi}$ smaller, the properties of Lemma 1 hold with respect to $\tilde{f}_{x}$ for all $y \in \mathbb{R}^{\mathrm{d}}$. Let $\Xi$ be the disjoint union given by $\Xi=\coprod_{x \in \Lambda^{*}}\{x\} \times \mathbb{R}^{\mathrm{d}}$ where $\Lambda^{*}$ is endowed with the discret topology. Then $\tilde{f}=\left(\tilde{f}_{x}\right)_{x \in \Lambda^{*}}$ can be viewed as a map from $\Xi$ to itself by letting $\tilde{f}(x, v)=\left(f(x), \tilde{f}_{x}(v)\right)$. Note that the global splitting $\coprod_{x \in \Lambda^{*}}\{x\} \times \mathbb{R}^{\mathrm{d}}=\coprod_{x \in \Lambda^{*}}\{x\} \times\left(E_{x}^{c s} \oplus E_{x}^{u}\right)$ is dominated with 
respect to $\tilde{f}$. By [16] $\S 5$, we can obtain a family $\left\{\mathcal{Y}_{x}^{c s}: x \in \Lambda^{*}\right\}$ of global $C^{1}$ submanifolds in $\mathbb{R}^{\mathrm{d}}$ which are $C^{1}$ graphs defined on $E_{x}^{c s}$ such that we have for all $x \in \Lambda^{*}$ :

$$
\begin{aligned}
& \{x\} \times \mathcal{Y}_{x}^{c s}=\bigcap_{n=0}^{+\infty} \tilde{f}^{-n}\left(\left\{f^{n}(x)\right\} \times Q_{\|\|_{x}^{\prime \prime}}\left(E_{f^{n}(x)}^{c s}, \xi\right)\right), \\
& \forall y \in \mathbb{R}^{\mathrm{d}}, T_{y} \mathcal{Y}_{x}^{c s} \subset Q_{\|\|_{x}^{\prime \prime}}\left(E_{x}^{c s}, \xi\right) .
\end{aligned}
$$

In particular we get $\tilde{f}^{ \pm}\left(\{x\} \times \mathcal{Y}_{x}^{c s}\right) \subset\left\{f^{ \pm}(x)\right\} \times \mathcal{Y}_{f^{ \pm}(x)}^{c s}$. Since we have $\left.\tilde{f}\right|_{\{x\} \times B\left(x, a_{\xi} \gamma_{\kappa(x)}\right)}=$ $\left.f\right|_{B\left(x, b_{\xi} \gamma_{\kappa(x)}\right)}$, one concludes the proof by considering $\mathcal{W}_{x}^{c s}=\mathcal{Y}_{x} \cap B\left(x, a_{\xi} \gamma_{\kappa(x)}\right)$ and taking much smaller $b_{\xi}$ than $a_{\xi}$.

\section{TAIL ENTROPY AND LOCAL VOLUME GROWTH}

Let $f: M \rightarrow M$ be a $\mathcal{C}^{r}$ diffeomorphism with $r>1$ on a compact Riemannian manifold $(M,\|\|)$. In this section, we relate the Newhouse local entropy of an ergodic measure with the local volume growth of smooth unstable discs. We begin with some definitions. A $\mathcal{C}^{r}$ map $\sigma$, from the unit square $[0,1]^{k}$ of $\mathbb{R}^{k}$ to $M$, which is a diffeomorphism onto its image, is called a $\mathcal{C}^{r}$ $k$-disc. The $\mathcal{C}^{r}$ size of $\sigma$ is defined as

$$
\|\sigma\|_{r}=\sup \left\{\left\|D^{q} \sigma\right\|: q \leq r, q \in \mathbb{R}^{+}\right\},
$$

where $\left\|D^{q} \sigma\right\|$ denotes the $(q-[q])$-Hölder norm of $D^{[q]} \sigma$ for $q \notin \mathbb{N}$ and the usual supremum norm of the derivative $D^{q} \sigma$ of order $q$ for $q \in \mathbb{N}$.

For any $\mathcal{C}^{1}$ smooth $k$-disc $\sigma$ and for any $\chi>0,1 \gg \gamma>0, C>1$ and $n \in \mathbb{N}$, we consider the set $\mathcal{H}_{f}^{n}(\sigma, \chi, \gamma, C)$ of points of $[0,1]^{k}$ whose exponential growth of the induced map on the $k$-exterior tangent bundle is almost equal to $\chi$ :

$\mathcal{H}_{f}^{n}(\sigma, \chi, \gamma, C):=\left\{t \in[0,1]^{k}: \forall 1 \leq j \leq n-1, C^{-1} e^{(\chi-\gamma) j} \leq\left\|\Lambda^{k} D_{t}\left(f^{j} \circ \sigma\right)\right\| \leq C e^{(\chi+\gamma) j}\right\}$.

For $\Gamma \subset[0,1]^{k}$, we also denote by $\left|\sigma_{\mid \Gamma}\right|$ the $k$-volume of $\sigma$ on $\Gamma$, i.e. $\left|\sigma_{\mid \Gamma}\right|=\int_{\Gamma}\left\|\Lambda^{k} D_{t} \sigma\right\| d \lambda(t)$, where $d \lambda$ is the Lebesgue measure on $[0,1]^{k}$. Then given $\chi>0,1 \gg \gamma>0, C>1, x \in M$, $n \in \mathbb{N}$ and $\varepsilon>0$, we define the local volume growth of $\sigma$ at $x$ with respect to these parameters as follows :

$$
V_{x}^{n, \varepsilon}(\sigma \mid \chi, \gamma, C):=\left|\left(f^{n-1} \circ \sigma\right)_{\mid \Delta_{n}}\right|
$$

with $\Delta_{n}:=\mathcal{H}_{f}^{n}(\sigma, \chi, \gamma, C) \cap \sigma^{-1} B_{n}(x, \varepsilon, f)$.

Proposition 2. Let $\nu \in \mathcal{M}_{\text {erg }}(f)$ with $l=l_{u}(f, \nu) \geq 1$. Then for any $\varepsilon>0,1>\eta>0$ and $\gamma>0$, there exist a Borel set $F_{\eta}$ with $\nu\left(F_{\eta}\right)>\eta$ and a constant $C>1$, such that for all $\delta>0$, all $n$ large enough and all $x \in F_{\eta}$ :

$$
s\left(n, \delta, B_{n}(x, \varepsilon, f) \cap F_{\eta}\right) \leq e^{\gamma n} \sup _{\substack{\sigma l d i s k \\ \text { with }\|\|_{r} \leq 1}} V_{x}^{n, 2 \varepsilon}\left(\sigma \mid \sum_{i} \lambda_{i}^{+}(\nu), \gamma, C\right) .
$$


In fact the $l_{u}$-discs can be chosen to be affine through the exponential map (see the proof of Proposotion 2 below). Let $v_{k}^{*}(f, \varepsilon)$ denote the local volume growth of $k$-disks :

$$
v_{k}^{*}(f, \varepsilon)=\limsup _{n} \frac{1}{n} \sup _{x \in M} \sup _{\substack{\sigma k-\text { disk } \\ \text { with }\|\sigma\|_{r} \leq 1}} \log \left|\left(f^{n-1} \circ \sigma\right)\right| \sigma^{-1} B_{n}(x, \varepsilon, f) \mid .
$$

S. Newhouse $[19,20]$ proved that the Newhouse local entropy $h^{*}(f, \nu, \varepsilon)$ of an ergodic measure is less than or equal to the local volume growth of center-unstable dimension. As a direct consequence of Proposition 2, we improve this estimate by considering the local volume growth of unstable dimension.

Corollary 1. With the above notations,

$$
\forall \varepsilon>0 \forall \nu \in \mathcal{M}_{e r g}(f), h^{*}(f, \nu, \varepsilon) \leq v_{l_{u}(f, \nu)}^{*}(f, 2 \varepsilon) .
$$

Such an inequality was established by K. Cogswell in [11] between the Kolmogorov-Sinai entropy and the global volume growth of unstable discs (in particular Cogswell's main result implies Corollary 1 for $\varepsilon$ larger than the diameter of $M$ ).

Remark 1. For any $\nu \in \mathcal{M}_{\text {erg }}(f)$, let us denote by $l_{c u}(f, \nu)$ the number of nonnegative Lyapunov exponents of $\nu$. The following estimate is shown in [20] :

$$
\forall \varepsilon>0 \forall \nu \in \mathcal{M}_{\text {erg }}(f), h^{*}(f, \nu, \varepsilon) \leq \sup _{\begin{array}{c}
\sigma l_{c u}(f, \nu)-\operatorname{disk} \\
\text { with }\|\sigma\|_{r} \leq 1
\end{array}} \limsup _{n} \frac{1}{n} \sup _{x \in M} \log \left|\left(f^{n-1} \circ \sigma\right)_{\mid \sigma^{-1} B_{n}(x, 2 \varepsilon, f)}\right| .
$$

Observe the right-hand side term differs from the local volume growth $v_{l_{c u}(f, \nu)}(f, 2 \varepsilon)$ as we invert the supremum in $\sigma$ with the limsup in $n$. We do not know if the above inequality still holds true for $l_{u}$ in place of $l_{c u}$.

We prove now Proposition 2 which is the key new tool to prove the existence of symbolic extensions in dimension 3 combining with the approach developed in [6].

Proof of Proposition 2. Consider $\nu \in \mathcal{M}_{\text {erg }}(f)$ with $l=l_{u}(f, \nu) \geq 1$. Let $0<\gamma \ll \lambda_{u}:=$ $\lambda_{l}(f, \nu)$ in the nonuniformly hyperbolic estimates of Section 2. Fix $\eta \in(0,1)$ and $k \in \mathbb{N}$ with $\nu\left(\Lambda_{k}\left(\lambda_{u}, \gamma\right)\right)>\eta$. There is a subset $F_{\eta}$ of $\Lambda_{k}=\Lambda_{k}\left(\lambda_{u}, \gamma\right)$ with $\nu\left(F_{\eta}\right)>\eta$ such that $\frac{1}{n}\left\|\Lambda^{l} D_{y}\left(\left.f^{n}\right|_{E_{y}^{u}}\right)\right\|$ is converging uniformly in $y \in F_{\eta}$ to $\sum_{i} \lambda_{i}^{+}(f, y)=\sum_{i} \lambda_{i}^{+}(f, \nu)$ when $n$ goes to $+\infty$. Let $\varepsilon \in(0,1)$ and $\varepsilon_{k}<\varepsilon$ to be precised. For any given $\hat{x} \in F_{\eta}, 0<\delta<\varepsilon$, let $E_{n}$ be a maximal $(n, \delta)$-separated set in $d$ for $f$ in $B_{n}(\hat{x}, \varepsilon, f) \cap F_{\eta}$. There exists $x \in E_{n}$ such that $E_{n}^{\prime}=E_{n} \cap B\left(x, \varepsilon_{k}\right)$ satisfies $\sharp E_{n}^{\prime} \geq A_{1}\left(\frac{\varepsilon_{k}}{\varepsilon}\right)^{\mathrm{d}} \sharp E_{n}$ for some universal constant $A_{1}$. Since we only deal with the local dynamics around the orbit of $x$, we can assume without loss of generality that we are working in $\mathbb{R}^{\mathrm{d}}$ by taking the exponential map at $x$. Take $0<\varepsilon_{k}<\left(a_{1} e^{k \gamma}\right)^{-1}$ so small that $B\left(x, \varepsilon_{k}\right) \subset B_{x}^{\prime \prime}\left(x, 2 a_{1} e^{k \gamma} \varepsilon_{k}\right) \subset B(x, \varepsilon)$ and consider

$$
\hat{\mathcal{W}}_{x}^{c s}=\left(x+E_{x}^{c s}\right) \cap B_{x}^{\prime \prime}\left(x, 2 a_{1} e^{k \gamma} \varepsilon_{k}\right) .
$$

For $\theta_{n}=\beta_{k} e^{-n\left(4 \gamma+l \gamma^{\prime}\right)}$ with $\beta_{k}=\beta_{k}(\delta)$ to be precised we let $\mathcal{A}^{c s}$ be a $\theta_{n}$-net of $\hat{\mathcal{W}}_{x}^{c s}$ for $d_{x}^{\prime \prime}$ satisfying $\sharp \mathcal{A}^{c s} \leq A_{2} \theta_{n}^{-\operatorname{dim} E^{c s}}=A_{2} \theta_{n}^{-(\mathrm{d}-l)}$ for some universal constant $A_{2}$. This means that any point of $\hat{\mathcal{W}}_{x}^{c s}$ is within a distance $\theta_{n}$ of $\mathcal{A}^{c s}$ for $d_{x}^{\prime \prime}$. For any $z \in \mathcal{A}^{c s}$, denote

$$
I_{z}=\left\{z+v:\|v\|_{x}^{\prime \prime} \leq 4 a_{1} e^{k \gamma} \varepsilon_{k}, v \in E_{x}^{u}\right\} .
$$


For $y \in B_{x}^{\prime \prime}\left(x, 2 a_{1} e^{k \gamma} \varepsilon_{k}\right)$ we let $y=y_{c s}+y_{u}$ with $y_{c s} \in x+E_{x}^{c s}$ and $y_{u} \in E_{x}^{u}$. Observe that $E_{x}^{c s}$ and $E_{x}^{u}$ are orthogonal in $<,>_{x}^{\prime \prime}$, thus $y_{c s}$ lies in $\hat{\mathcal{W}}_{x}^{c s}$ and there exists $z_{y} \in \mathcal{A}^{c s}$ with $\left\|y_{c s}-z_{y}\right\|_{x}^{\prime \prime}<\theta_{n}$. Therefore, when $y$ also lies in $\Lambda_{k}$ we get :

$$
\begin{aligned}
\left\|y_{c s}-z_{y}\right\|_{y}^{\prime \prime} & \leq 2 a_{1} e^{k \gamma}\left\|y_{c s}-z_{y}\right\| \\
& \leq 4 a_{1} e^{k \gamma}\left\|y_{c s}-z_{y}\right\|_{x}^{\prime \prime} \\
& \leq 4 a_{1} e^{k \gamma} \theta_{n} .
\end{aligned}
$$

For small $\xi \in\left(0, \frac{1}{4}\right)$, let $b_{\xi}>0$ be as in Lemma 1 and Proposition 1. Since the distributions $E^{c s}$ and $E^{u}$ are continuous on $\Lambda_{k}$, we may choose $\varepsilon_{k}$ and $\beta_{k}$ so small that for any $y \in E_{n}^{\prime}$ :

- the set $\left(\left[y_{c s}, z_{y}\right]+E_{x}^{u}\right) \cap \mathcal{W}_{y}^{c s}$ defines a graph $\Gamma_{\phi_{y}}$ of a $C^{1}$ function $\phi_{y}:\left[y_{c s}, z_{y}\right] \subset E_{x}^{c s} \rightarrow$ $E_{x}^{u}$

- $E_{x}^{c s / u} \subset Q_{\|\| \|}\left(E_{y}^{c s / u}, \frac{\xi}{4 a_{1} e^{k \gamma}}\right) \subset Q_{\|\|_{y}^{\prime \prime}}\left(E_{y}^{c s / u}, \xi\right)$, these cones being defined with respect to the splitting $E_{y}^{c s} \oplus E_{y}^{u}$.

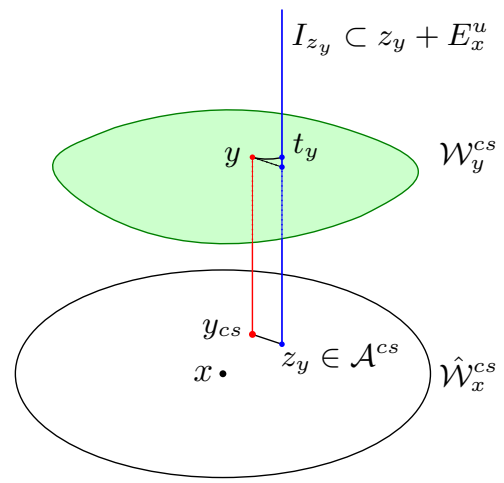

FiguRE 1. The transverse intersection at $t_{y}$ of $I_{z_{y}}$ and $\mathcal{W}_{y}^{c s}$ for $y \in E_{n}^{\prime}$.

Let $\theta_{y}:[0,1] \rightarrow E_{x}^{c s}+E_{x}^{u}$ be the reparametrization of the graph of $\phi_{y}$ given by

$$
\forall t \in[0,1], \theta_{y}(t)=y_{c s}+t\left(z_{y}-y_{c s}\right)+\phi\left(y_{c s}+t\left(z_{y}-y_{c s}\right)\right. \text {. }
$$

Note that $\theta_{y}(0)=y$ and $\theta_{y}(1)$ is the intersection point of $I_{z_{y}}$ and $\mathcal{W}_{y}^{c s}$. To simplify the notations we let $t_{y}:=\theta_{y}(1)$. It follows from the almost tangency property of center-stable fake manifolds stated in Proposition 1 (ii) that

$$
\theta^{\prime}(t) \in Q_{\|\|_{y}^{\prime \prime}}\left(E_{y}^{c s}, \xi\right)
$$

Moreover we have

$$
\begin{aligned}
& z_{y}-y_{c s} \in E_{x}^{c s} \subset Q_{\|\|_{y}^{\prime \prime}}\left(E_{y}^{c s}, \xi\right), \\
& D_{y_{c s}+t\left(z_{y}-y_{c s}\right)} \phi\left(z_{y}-y_{c s}\right) \quad \in E_{x}^{u} \subset Q_{\|\|_{y}^{\prime \prime}}\left(E_{y}^{u}, \xi\right) .
\end{aligned}
$$

From the above properties (7), (8), (9) and $\xi<\frac{1}{4}$, one deduces after an easy computation that $\left\|\theta^{\prime}(t)\right\|_{y}^{\prime \prime} \leq 3\left\|z_{y}-y_{c s}\right\|_{y}^{\prime \prime}$ for all $t \in[0,1]$. For $w \in \Lambda^{*}$ let $d_{\mathcal{W}_{w}^{c s}}^{\prime \prime}$ be the distance induced 
respectively by \|\|$_{w}^{\prime \prime}$ on $\mathcal{W}_{w}^{c s}$. We have

$$
\begin{aligned}
d_{\mathcal{W}_{y}^{c s}}^{\prime \prime}\left(y, t_{y}\right) & \leq \int_{[0,1]}\left\|\theta^{\prime}(t)\right\|_{y}^{\prime \prime} d t \\
& \leq 3\left\|y_{c s}-z_{y}\right\|_{y}^{\prime \prime} \\
& \leq 12 a_{1} e^{k \gamma} \theta_{n}
\end{aligned}
$$

Consequently by the local invariance of center-stable manifolds stated in Proposition 1 (iii) we get for all $0<j \leq n$ :

$$
d_{\mathcal{W}_{f^{j}(y)}^{c s}}^{\prime \prime}\left(f^{j}(y), f^{j}\left(t_{y}\right)\right) \leq\left\|\left.D f\right|_{T \mathcal{W}_{f^{j-1}(y)}^{c s}}\right\|_{f^{j-1}(y)}^{\prime \prime} d_{\mathcal{W}_{f^{j-1}(y)}^{c s}}^{\prime \prime}\left(f^{j-1}(y), f^{j-1}\left(t_{y}\right)\right)
$$

and then by Lemma 1 (i),

$$
d_{\mathcal{W}_{f^{j}(y)}^{c s}}^{\prime \prime}\left(f^{j}(y), f^{j}\left(t_{y}\right)\right) \leq e^{4 \gamma} d_{\mathcal{W}_{f^{j-1}(y)}^{c s}}^{\prime \prime}\left(f^{j-1}(y), f^{j-1}\left(t_{y}\right)\right)
$$

After an immediate induction we obtain for all $0 \leq j \leq n$ :

$$
d_{\mathcal{W}_{f^{j}(y)}^{c s}}^{\prime \prime}\left(f^{j}(y), f^{j}\left(t_{y}\right)\right) \leq e^{4 j \gamma} d_{\mathcal{W}_{y}^{c s}}^{\prime \prime}\left(y, t_{y}\right)
$$

and therefore

$$
\begin{aligned}
d_{\mathcal{W}_{f^{j}(y)}^{c s}}^{\prime \prime}\left(f^{j}(y), f^{j}\left(t_{y}\right)\right) & \leq 12 a_{1} e^{k \gamma} e^{4 n \gamma} \theta_{n} \\
& \leq 12 a_{1} e^{k \gamma} \beta_{k} e^{-n l \gamma^{\prime}}, \\
d_{\mathcal{W}_{f^{j}(y)}^{c s}}^{\prime \prime}\left(f^{j}(y), f^{j}\left(t_{y}\right)\right) & \leq 12 a_{1} e^{k \gamma} \frac{\beta_{k}}{\gamma_{\kappa(y)}} \gamma_{\kappa\left(f^{j}(y)\right)} .
\end{aligned}
$$

As $y$ belongs to $E_{n}^{\prime} \subset \Lambda_{k}$ we have $\kappa(y) \leq k$. Therefore we get for $\beta_{k} \leq \frac{b_{\xi} \gamma_{k}}{48 a_{1} e^{k \gamma}}$ :

$$
\begin{aligned}
\forall 0 \leq j \leq n, d\left(f^{j}\left(t_{y}\right), f^{j}(y)\right) & \leq 2 d_{\mathcal{W}_{f^{j}(y)}^{c s}}^{\prime \prime}\left(f^{j}(y), f^{j}\left(t_{y}\right)\right) \\
& \leq \frac{b_{\xi}}{2} \gamma_{\kappa\left(f^{j}(y)\right)},
\end{aligned}
$$

and we have similarly for $\beta_{k}<\frac{\delta}{48 a_{1} e^{k \gamma}}$ :

$$
\begin{gathered}
\forall 0 \leq j \leq n, d\left(f^{j}\left(t_{y}\right), f^{j}(y)\right) \leq \delta / 4, \\
\text { i.e. } t_{y} \in B_{n}(y, \delta / 4, f) .
\end{gathered}
$$

For $y \in E_{n}^{\prime}$ we let now

$$
\begin{aligned}
W_{n}\left(t_{y}\right) & :=\bigcap_{j=0}^{n-1} f^{-j}\left(B_{f^{j}(y)}^{\prime \prime}\left(f^{j}\left(t_{y}\right), \frac{\delta}{8} e^{-l j \gamma^{\prime}}\right)\right) \bigcap I_{z}, \\
& \subset B_{n}\left(t_{y}, \delta / 4, f\right), \\
& \subset B_{n}(y, \delta / 2, f) .
\end{aligned}
$$

As $E_{n}^{\prime}$ is $(n, \delta)$-separated, the sets $\left(W_{n}\left(t_{y}\right)\right)_{y \in E_{n}^{\prime}}$ are pairwise disjoint. 
For $\delta$ small enough (depending only on $k$ ), for any $j=0, \cdots, n-1$, the ball $B_{f^{j}(y)}^{\prime \prime}\left(f^{j}\left(t_{y}\right), \frac{\delta}{8} e^{-l j \gamma^{\prime}}\right)$ is contained in $B\left(f^{j}(y), b_{\xi} \gamma_{\kappa\left(f^{j}(y)\right)}^{l}\right)$, since $d\left(f^{j}\left(t_{y}\right), f^{j}(y)\right) \leq \frac{b_{\xi}}{2} \gamma_{\kappa\left(f^{j}(y)\right)}^{l}$. Let $\left(e_{x}^{i}\right)$ be an orthonormal basis of $E_{x}^{u}$ with respect to $\|\cdot\|_{x}^{\prime \prime}$. We consider the affine reparametrization of $I_{z}, z \in \mathcal{A}^{c s}$, given by $\sigma_{z}:[0,1]^{l_{\nu}} \rightarrow M,\left(t_{i}\right)_{i} \mapsto z+\sum_{i}\left(t_{i}-1 / 2\right) 4 a_{1} e^{k \gamma} \varepsilon_{k} e_{x}^{i}$. Noting that $E_{x}^{u} \in Q_{\|\|_{y}^{\prime \prime}}\left(E_{y}^{u}, \xi\right)$, by Lemma 1 (ii), for any $\tau \in \sigma_{z}^{-1} W_{n}\left(t_{y}\right)$ and for any $0 \leq j \leq n$, the vector space $D_{\sigma_{z}(\tau)} f^{j}\left(E_{x}^{u}\right)$ lies in $Q_{\|\|_{f^{j}(y)}^{\prime \prime}}\left(E_{f^{j}(y)}^{u}, \xi\right)$. Then by Lemma 1 (iii) we get

$$
\begin{aligned}
\limsup _{n} \frac{1}{n} \log \left\|\left.\Lambda^{l} D_{\sigma_{z}(\tau)} f^{n}\right|_{E_{x}^{u}}\right\|_{y}^{\prime \prime} & =\limsup _{n} \frac{1}{n} \sum_{j=0}^{n-1} \log \left\|\left.\Lambda^{l} D_{f^{j} \circ \sigma_{z}(\tau)} f\right|_{D_{\sigma_{z}(\tau)} f^{j}\left(E_{x}^{u}\right)}\right\|_{f^{j}(y)}^{\prime \prime}, \\
& \leq \underset{n}{\limsup } \frac{1}{n} \sum_{j=0}^{n-1} \log \left\|\left.\Lambda^{l} D_{f^{j}(y)} f\right|_{E_{f^{j}(y)}^{u}}\right\|_{f^{j}(y)}^{\prime \prime}+\gamma, \\
& =\limsup _{n} \frac{1}{n} \log \left\|\left.\Lambda^{l} D_{y} f^{n}\right|_{E_{y}^{u}}\right\|_{y}^{\prime \prime}+\gamma .
\end{aligned}
$$

Noting that $f^{n}(y) \in \Lambda_{k+n}$, we have by the Inequalities (4)

$$
\forall v \in T_{f^{n}\left(\sigma_{z}(\tau)\right)} M, \frac{\|v\|}{2} \leq\|v\|_{f^{n}(y)}^{\prime \prime} \leq 2 a_{1} e^{(k+n) \gamma}\|v\| .
$$

Then it follows from Lemma 2 in the Appendix that

$$
\begin{aligned}
\limsup _{n} \frac{1}{n} \log \left\|\Lambda^{l} D_{\tau}\left(f^{n} \circ \sigma_{z}\right)\right\| & =\underset{n}{\limsup } \frac{1}{n} \log \left\|\left.\wedge^{l} D_{\sigma_{z}(\tau)} f^{n}\right|_{E_{x}^{u}}\right\|, \\
& \leq \underset{n}{\limsup } \frac{1}{n} \log \left\|\left.\Lambda^{l} D_{\sigma_{z}(\tau)} f^{n}\right|_{E_{x}^{u}}\right\|_{y}^{\prime \prime}+l \gamma, \\
& \leq \underset{n}{\limsup } \frac{1}{n} \log \left\|\wedge^{l}\left(\left.D_{y} f^{n}\right|_{E_{y}^{u}}\right)\right\|_{y}^{\prime \prime}+(l+1) \gamma, \\
& \leq \limsup _{n} \frac{1}{n} \log \left\|\wedge^{l}\left(\left.D_{y} f^{n}\right|_{E_{y}^{u}}\right)\right\|+(2 l+1) \gamma, \\
& =\sum_{i} \lambda_{i}^{+}(f, \nu)+(2 l+1) \gamma .
\end{aligned}
$$

Similarly we also get :

$$
\liminf _{n} \frac{1}{n} \log \left\|\wedge^{l} D_{\tau}\left(f^{n} \circ \sigma_{z}\right)\right\| \geq \sum_{i} \lambda_{i}^{+}(f, \nu)-(2 l+1) \gamma .
$$

Moreover the above limsup and liminf are uniform in $y \in E_{n}^{\prime}$ and $\tau \in \sigma_{z}^{-1} W_{n}\left(t_{y}\right)$. Therefore for some $C>1$ we have for $n$ large enough,

$$
\sigma_{z}^{-1} W_{n}\left(t_{y}\right) \subset \mathcal{H}_{f}^{n}\left(\sigma_{z}, \sum_{i} \lambda_{i}^{+}(f, \nu),(2 l+2) \gamma, C\right) .
$$

By using Lemma 1 and classical arguments of graph transform, the set $f^{j}\left(W_{n}\left(t_{y}\right)\right)$ for $0 \leq j \leq$ $n-1$ defines a graph of a function from $\left.B_{f^{j}(y)}^{\prime \prime}\left(f^{j}\left(t_{y}\right), \frac{\delta}{8} e^{-l j \gamma^{\prime}}\right)\right) \cap E_{f^{j}(y)}^{u}$ to $E_{f^{j}(y)}^{c s}$. Therefore 
the $l$-volume of $f^{n-1}\left(W_{n}\left(t_{y}\right)\right)$ with respect to $\|\cdot\|_{f^{n-1}(y)}^{\prime \prime}$ satisfies

$$
\left|f^{n-1}\left(W_{n}\left(t_{y}\right)\right)\right|_{f^{n-1}(y)}^{\prime \prime} \geq c_{l} \delta^{l} e^{-l^{2}(n-1) \gamma^{\prime}},
$$

for some universal constant $c_{l}$. By applying again Lemma 2 in the Appendix we obtain :

$$
\left|f^{n-1}\left(W_{n}\left(t_{y}\right)\right)\right| \geq\left(4 a_{1} e^{(k+n-1) \gamma}\right)^{-l}\left|f^{n-1}\left(W_{n}\left(t_{y}\right)\right)\right|_{f^{n-1}(y)}^{\prime \prime},
$$

where $\left|f^{n-1}\left(W_{n}\left(t_{y}\right)\right)\right|$ denotes the $l$-volume of $f^{n-1}\left(W_{n}\left(t_{y}\right)\right)$ with respect to the Riemannian norm $\|\cdot\|$ on $M$. For $z \in \mathcal{A}^{c s}$ we let

$$
\Gamma_{z}:=\left\{y \in E_{n}^{\prime}, z_{y}=z\right\}
$$

and

$$
\Delta_{n}^{z}:=\mathcal{H}_{f}^{n}\left(\sigma_{z}, \sum_{i} \lambda_{i}^{+}(f, \nu),(2 l+2) \gamma, C\right) \cap \sigma_{z}^{-1} B_{n}(x, 2 \varepsilon, f) .
$$

As the sets $W_{n}\left(t_{y}\right), y \in \Gamma_{z}$, are pairwise disjoint subsets of $\sigma_{z}\left(\Delta_{n}^{z}\right)$ we have :

$$
\left|\left(f^{n-1} \circ \sigma_{z}\right)_{\mid \Delta_{n}}\right| \geq \sum_{y \in \Gamma_{z}}\left|f^{n-1}\left(W_{n}\left(t_{y}\right)\right)\right| .
$$

By combining the inequalities (10), (11), (12) we obtain

$$
\begin{aligned}
\left|\left(f^{n-1} \circ \sigma_{z}\right)_{\mid \Delta_{n}^{z}}\right| & \geq\left(4 a_{1} e^{(k+n-1) \gamma}\right)^{-l} \sum_{y \in \Gamma_{z}}\left|f_{\mid W_{n}\left(t_{y}\right)}^{n-1}\right|_{f^{n-1}(y)}^{\prime \prime}, \\
& \geq c_{l} \delta^{l} e^{-l^{2}(n-1) \gamma^{\prime}}\left(4 a_{1} e^{(k+n-1) \gamma}\right)^{-l} \cdot \sharp \Gamma_{z} .
\end{aligned}
$$

With the notations introduced at the beginning of Section 3, we have therefore for some constant $D$ independent of $n$ and $\hat{x} \in F_{\eta}$,

$$
\# \Gamma_{z} \leq D e^{n\left(l \gamma+l^{2} \gamma^{\prime}\right)} V_{x}^{n, 2 \varepsilon}\left(\sigma_{z} \mid \sum_{i} \lambda_{i}^{+}(f, \nu),(2 l+2) \gamma, C\right) .
$$

By letting $\mathcal{F}_{n, \delta}:=\left\{\sigma_{z}, z \in \mathcal{A}^{c s}\right\}$, we get for all $\hat{x} \in \Lambda_{k}$ and some constants, all denoted by $D$ and independent of $n$ and $\hat{x} \in F_{\eta}$ :

$$
\begin{aligned}
s\left(n, \delta, B_{n}(\hat{x}, \varepsilon, f)\right) & =\sharp E_{n}, \\
& \leq D \sharp E_{n}^{\prime}, \\
& \leq D \sum_{z \in \mathcal{A}^{c s}} \sharp \Gamma_{z}, \\
& \leq D e^{n\left(l \gamma+l^{2} \gamma^{\prime}\right) \sharp \mathcal{A}^{c s}} \sup _{\sigma \in \mathcal{F}_{n, \delta}} V_{x}^{n, 2 \varepsilon}\left(\sigma_{z} \mid \sum_{i} \lambda_{i}^{+}(f, \nu),(2 l+2) \gamma, C\right), \\
& \leq D e^{n\left((\mathrm{~d}-l)\left(4 \gamma+l \gamma^{\prime}\right)+l \gamma+l^{2} \gamma^{\prime}\right)} \sup _{\sigma \in \mathcal{F}_{n, \delta}} V_{x}^{n, 2 \varepsilon}\left(\sigma_{z} \mid \sum_{i} \lambda_{i}^{+}(f, \nu),(2 l+2) \gamma, C\right) .
\end{aligned}
$$

This concludes the proof of Proposition 2 as $\gamma$ and thus $\gamma^{\prime}=\alpha^{-1} \gamma$ may be chosen arbitrarily small. 


\section{Proof of Main Theorem}

By Proposition 2 Newhouse local entropy of an ergodic measure with one positive Lyapunov exponent is bounded from above by the local volume growth of curves. This volume growth may be controled by using the Reparametrization Lemma of [6]. Following straightforwardly the proof of the Main Proposition in [6] we get :

Proposition 3. Let $f$ be a $\mathcal{C}^{r}$ diffeomorphism with $r>1$ on a Riemannian manifold $M$ and $\mu \in \mathcal{M}_{\text {inv }}(f)$. For all $\gamma>0$, there exist $m_{\mu}, k_{\mu} \in \mathbb{N}^{*}$ such that for $\nu \in \mathcal{M}_{\text {erg }}(f)$ close enough to $\mu$ with $l_{u}(f, \nu)=1$, we have

$$
h_{m_{\mu}, k_{\mu}}^{N e w}(f, \nu) \leq \frac{\lambda_{1}^{+}(f, \mu)-\lambda_{1}^{+}(f, \nu)}{r-1}+\gamma .
$$

From the criterion in Theorem 1, for proving the Main Theorem, we need consider all ergodic measures with any possible $l_{u}$. Actually, the Main Theorem is obtained from the following Proposition by applying Theorem 1 with the upper semicontinuous affine function $E:=\frac{1}{r-1} \sum_{i=1,2} \lambda_{i}^{+}(f, \cdot)$.

Proposition 4. Let $f$ be a $\mathcal{C}^{r}$ diffeomorphism with $r>1$ on a 3-dimensional Riemannian manifold $M$ and $\mu \in \mathcal{M}_{\text {inv }}(f)$. For all $\gamma>0$, there exist an entropy structure $\left(h_{k}\right)_{k}$ and $k_{\mu} \in \mathbb{N}$ such that for $\nu \in \mathcal{M}_{\text {erg }}(f)$ close enough to $\mu$, we have

$$
h_{k_{\mu}}(f, \nu) \leq \frac{\sum_{i=1,2} \lambda_{i}^{+}(f, \mu)-\sum_{i=1,2} \lambda_{i}^{+}(f, \nu)}{r-1}+\gamma .
$$

In other terms, $E:=\frac{1}{r-1} \sum_{i=1,2} \lambda_{i}^{+}(f, \cdot)$ satisfies Inequaliy (1) for a 3 -dimensional $\mathcal{C}^{r}$ diffeomorphism $f$ with $r>1$.

Proof of Proposition 4. Fix $\mu \in \mathcal{M}_{\text {inv }}(f)$. By the upper semicontinuity of $\sum_{i=1,2} \lambda_{i}^{+}(f, \cdot)$, lower semicontinuity of $\lambda_{3}(f, \cdot)$ and continuity of the integral of logarithm for Jacobian, when $\nu$ is close enough to $\mu$, one has

$$
\begin{aligned}
\sum_{i=1,2} \lambda_{i}^{+}(f, \mu)-\sum_{i=1,2} \lambda_{i}^{+}(f, \nu) & \geq-\frac{(r-1) \gamma}{2}, \\
\lambda_{3}(f, \mu)-\lambda_{3}(f, \nu) & \leq \frac{\gamma}{2}, \\
\left|\int \log \operatorname{Jac}(f) d \nu-\int \log \operatorname{Jac}(f) d \mu\right| & \leq(r-1) \gamma .
\end{aligned}
$$

Hence, if $h_{\nu}(f) \leq \gamma / 2$, from $h_{m, k}^{N e w}(f, \nu) \leq h_{\nu}(f)$ for any $m, k$, by (13) we get

$$
h_{m, k}^{N e w}(f, \nu) \leq \frac{\sum_{i=1,2} \lambda_{i}^{+}(f, \mu)-\sum_{i=1,2} \lambda_{i}^{+}(f, \nu)}{r-1}+\gamma .
$$

Next we assume $h_{\nu}(f)>\gamma / 2$. By Ruelle inequality [23], it holds that $\min \left(l_{u}(f, \nu), l_{u}\left(f^{-1}, \nu\right)\right)=$ 1. Applying Proposition 3 to $f^{ \pm}$, there exist $m_{\mu}^{ \pm}, k_{\mu}^{ \pm} \in \mathbb{N}$ such that for any $\nu \in \mathcal{M}_{\text {erg }}(f)$ close enough to $\mu$ with $l_{u}\left(f^{ \pm}, \nu\right)=1$,

$$
h_{m_{\mu}^{ \pm}, k_{\mu}^{ \pm}}^{N e w}\left(f^{ \pm}, \nu\right) \leq \frac{\lambda_{1}^{+}\left(f^{ \pm}, \mu\right)-\lambda_{1}^{+}\left(f^{ \pm}, \nu\right)}{r-1}+\gamma .
$$


If $l_{u}(f, \nu)=1$, then $\sum_{i=1,2} \lambda_{i}^{+}(f, \nu)=\lambda_{1}^{+}(f, \nu)$, thus by the above inequality, (16) holds with respect to $m_{\mu}^{+}, k_{\mu}^{+}$. If $l_{u}\left(f^{-1}, \nu\right)=1$, then $\lambda_{3}(f, \nu) \leq-h_{\nu}\left(f^{-1}\right)=-h_{\nu}(f)<-\gamma / 2$, which implies $\lambda_{3}(f, \mu)<0$ by (14). Thus,

$$
\begin{aligned}
\lambda_{1}^{+}\left(f^{-1}, \mu\right)-\lambda_{1}^{+}\left(f^{-1}, \nu\right) & =\lambda_{3}^{-}(f, \nu)-\lambda_{3}^{-}(f, \mu) \\
& =\lambda_{3}(f, \nu)-\lambda_{3}(f, \mu) .
\end{aligned}
$$

Noting that $\int \log \operatorname{Jac}(f) d \tau=\sum_{i=1,2,3} \lambda_{i}(f, \tau)$ for any $\tau \in \mathcal{M}_{i n v}(f)$, by (15) we finally get

$$
\begin{aligned}
\lambda_{1}^{+}\left(f^{-1}, \mu\right)-\lambda_{1}^{+}\left(f^{-1}, \nu\right) & =\sum_{i=1,2} \lambda_{i}(f, \mu)-\sum_{i=1,2} \lambda_{i}(f, \nu)+\int \log \operatorname{Jac}(f) d \nu-\int \log \operatorname{Jac}(f) d \mu \\
& \leq \sum_{i=1,2} \lambda_{i}^{+}(f, \mu)-\sum_{i=1,2} \lambda_{i}^{+}(f, \nu)+(r-1) \gamma
\end{aligned}
$$

and therefore

$$
h_{m_{\mu}^{-}, k_{\mu}^{-}}^{N e w}\left(f^{-1}, \nu\right) \leq \frac{\sum_{i=1,2} \lambda_{i}^{+}(f, \mu)-\sum_{i=1,2} \lambda_{i}^{+}(f, \nu)}{r-1}+\gamma .
$$

By Lemma 2 in [6], the sequence $\left(\underline{h_{k}}\right)_{k}:=\left(\min \left(h_{m_{\mu}^{+}, k}^{N e w}(f, \cdot), h_{m_{\mu}^{-}, k}^{N e w}\left(f^{-1} \cdot\right)\right)\right)$ defines an entropy structure. Combining (16) for $l_{u}(f, \nu)=1$ and (17) for $l_{u}\left(f^{-1}, \nu\right)=1$, we conclude the proof by considering the entropy structure $\left(\underline{h_{k}}\right)_{k}$.

Remark 2. For a local diffeomorphism $f: M \rightarrow M$, the following local Ruelle inequlity holds [7][10] : there exists a scale $\varepsilon>0$ such that $h^{*}(f, \mu, \varepsilon) \leq \min \left(\sum_{j} \lambda_{j}^{+}(f, \mu),-\sum_{j} \lambda_{j}^{-}(f, \mu)\right)$ for any $\mu \in \mathcal{M}_{\text {inv }}(f)$. In particular in dimension 3 , any invariant measure with positive Newhouse local entropy admits at least one positive and one negative Lyapunov exponent. As the proofs of Main Theorem and Proposition 2 are just local they apply verbatim in the context of local 3-dimensional diffeomorphism.

\section{Appendix A.}

Let $E$ and $F$ be two finite dimensional vector spaces of dimension $k$. We endow $E$ (resp. $F$ ) with two Euclidean norms $\|\cdot\|_{E}$ and $\|\cdot\|_{E}^{\prime}$ (resp. $\|\cdot\|_{F}$ and $\|\cdot\|_{F}^{\prime}$ ). We consider the associated Euclidean structures on $\curlywedge^{k} E\left(\right.$ resp. $\left.\curlywedge^{k} F\right)$. Let $A: E \rightarrow F$ be an invertible linear map and $\curlywedge^{k} A$ the induced map on the $k$-exterior powers. We denote by $\left\|\curlywedge^{k} A\right\|$ and $\left\|\wedge^{k} A\right\|^{\prime}$ the associated subordinated norms.

Lemma 2. With the above notations. Assume that we have for some constants $C_{E}, C_{F} \geq 1$ and $D_{E}, D_{F} \leq 1$ :

$$
\begin{aligned}
& \forall v \in E, \quad D_{E}\|v\|_{E} \leq\|v\|_{E}^{\prime} \leq C_{E}\|v\|_{E}, \\
& \forall w \in F, \quad D_{F}\|w\|_{F} \leq\|w\|_{F}^{\prime} \leq C_{F}\|w\|_{F},
\end{aligned}
$$

then

$$
\left(D_{F} / C_{E}\right)^{k}\left\|\curlywedge^{k} A\right\| \leq\left\|\curlywedge^{k} A\right\|^{\prime} \leq\left(C_{F} / D_{E}\right)^{k}\left\|\Lambda^{k} A\right\| .
$$

Proof. By the singular value decomposition there exists an orthonormal family $\left(e_{i}\right)_{i=1, \cdots, k}$ of $\left(E,\|\cdot\|_{E}\right)$ such that $\left(A e_{i}\right)_{i}$ is an orthogonal family in $\left(F,\|\cdot\|_{F}\right)$ with $\left\|{ }^{k} A\right\|=\left\|A e_{1} \cdots \wedge A e_{k}\right\|_{F}=$ $\prod_{i=1}^{k}\left\|A e_{i}\right\|_{F}$. Similarly we let $\left(e_{i}^{\prime}\right)_{i=1, \cdots, k}$ be the corresponding orthonormal family for the 
norms $\|\cdot\|_{E}^{\prime}$ and $\|\cdot\|_{F}^{\prime}$. Let $P$ be the change of basis matrix from $\left(e_{i}^{\prime}\right)_{i}$ to $\left(e_{i}\right)_{i}$. Then the norms $\left\|e_{1} \wedge \cdots \wedge e_{k}\right\|_{E}^{\prime}$ and $\left\|e_{1}^{\prime} \wedge \cdots \wedge e_{k}^{\prime}\right\|_{E}$ are just given by the absolute values of the determinants of $P$ and $P^{-1}$ respectively. Therefore we have

$$
\begin{aligned}
\left|\operatorname{det}\left(P^{-1}\right)\right| & \leq \prod_{i}\left\|e_{i}^{\prime}\right\|_{E} \\
& \leq D_{E}^{-k},
\end{aligned}
$$

and

$$
\begin{aligned}
\left\|e_{1} \wedge \cdots \wedge e_{k}\right\|_{E}^{\prime} & =|\operatorname{det}(P)| \\
& =1 /\left|\operatorname{det}\left(P^{-1}\right)\right| \\
& \geq D_{E}^{k} .
\end{aligned}
$$

We conclude that

$$
\begin{aligned}
\left\|\wedge^{k} A\right\|^{\prime} & \leq \frac{\left\|A e_{1} \wedge \cdots \wedge A e_{k}\right\|_{F}^{\prime}}{\left\|e_{1} \wedge \cdots \wedge e_{k}\right\|_{E}^{\prime}} \\
& \leq D_{E}^{-k} \prod_{i}\left\|A e_{i}\right\|_{F}^{\prime} \\
& \leq D_{E}^{-k} C_{F}^{k} \prod_{i}\left\|A e_{i}\right\|_{F} \\
& \leq\left(C_{F} / D_{E}\right)^{k}\left\|\wedge^{k} A\right\| .
\end{aligned}
$$

The other inequality is obtained symmetrically.

\section{REFERENCES}

[1] M. Asaoka, Hyperboic sets exhibiting $C^{1}$-persistent homoclonic tangency for higher dimensions, Proc. Amer. Math. Soc., 136, 677-686, 2008. 1

[2] L. Barreira and Y. Pesin, Nonuniform hyperbolicity: Dynamics of systems with nonzero Lyapunov exponents, Cambridge Press, 2007. 4

[3] M. Boyle and T. Downarowicz, The entropy theory of symbolic extensions, Invent. Math., 156, 119-161, 2004. 1, 2

[4] M. Boyle, D. Fiebig and U. Fiebig, Residual entropy, conditional entropy and subshift covers, Forum Math., 14, , 713-757, 2002. 1

[5] D. Burguet, $\mathcal{C}^{2}$ surface diffeomorphisms have symbolic extensions, Invent. Math., 186, 191-236, 2011. 1

[6] D. Burguet, Symbolic extensions in intermediate smoothness on surfaces, Ann. Sci. Éc. Norm. Supér., 2, 337-362, 2012. 1, 2, 3, 9, 14, 15

[7] D. Burguet and T. Fisher, Symbolic extensions for partially hyperbolic dynamical systems with 2dimensional center bundle, Discrete Contin. Dyn. Syst., 33, 2253-2270, 2013. 15

[8] J. Buzzi, Intrinsic ergodicity for smooth interval maps, Israel J. Math., 100, 125-161, 1997. 1

[9] J. Buzzi, S. Crovisier and T. Fisher, The entropy of $C^{1}$-diffeomorphisms without a dominated splitting, Trans. Amer. Math. Soc., 370, 6685-6734, 2018. 1

[10] Y. Cao, G. Liao and Z. You, Upper bounds on measure theoretic tail entropy for dominated splittings, Ergodic Theory Dynam. Systems., arXiv:1901.01694. 15

[11] K. Cogswell, Entropy and volume growth, Ergodic Theory Dynam. Systems., 20,. 77-84, 2000. 9

[12] W. Cowieson and L. S. Young, SRB measures as zero-noise limits, Ergodic Theory Dynam. Systems., 25, 1115-1138, 2005. 1 
[13] T. Downarowicz, Entropy structure, J. Anal. Math., 96, 57-116, 2005. 2, 3

[14] T. Downarowicz and A. Maass, Smooth interval maps have symbolic extensions, Invent. Math., 176, 617636, 2009. 1, 2

[15] T. Downarowicz and S. Newhouse, Symbolic extension entropy in smooth dynamics, Invent. Math., 160, 453-499, 2005. 1

[16] M. Hirsch, C. Pugh and M. Shub, Invariant manifolds, volume 583 of Lect. Notes in Math., Springer Verlag, 1977. 8

[17] G. Liao, M. Viana and J. Yang, The entropy conjecture for diffeomorphisms away from tangencies, J. Eur. Math. Soc., 15, 2043-2060, 2013. 1

[18] L. Díaz, T. Fisher, M. J. Pacifico and J. Vieitez, Entropy-expansiveness for partially hyperbolic diffeomorphisms, Discrete Con. Dyn. Syst., 32, 4195-4207, 2012. 1

[19] S. Newhouse, Entropy and volume, Ergodic Theory Dynam. Systems., 8*, 283-299, 1988. 9

[20] S. Newhouse, Continuity properties of entropy, Ann. Math., 129, 215-235, 1989. 2, 9

[21] V. I. Oseledets, A multiplicative ergodic theorem, Trans. Moscow Math. Soc., 19, 197-231, 1968. 3

[22] M. Pollicott, Lectures on ergodic theory and Pesin theory on compact manifolds, Cambridge Univ. Press, Cambridge, 1993. 4, 5

[23] D. Ruelle, An inequality for the entropy of differentiable maps, Bol. Soc. Bras. Mat., 9, 83-88, 1978. 14

[24] Y. Yomdin, Volume growth and entropy, Israel J. Math., 57, 285-300, 1987. 1

E-mail address: david.burguet@upmc.fr

E-mail address: lg@suda.edu.cn 